\title{
Image-Based Quantification of Cell Debris as a Measure of Apoptosis
}

\author{
Magnus Ölander, ${ }^{\dagger}$ Ni Niklas Handin, ${ }^{\dagger}$ and Per Artursson $*,+$ \\ ${ }^{\dagger}$ Department of Pharmacy, Uppsala University, SE-75123 Uppsala, Sweden \\ ${ }^{\star}$ Department of Pharmacy and Science for Life Laboratory, Uppsala University, SE-75123 Uppsala, Sweden
}

\section{Supporting Information}

ABSTRACT: Apoptosis is a controlled form of cell death that can be induced by various diseases and exogenous toxicants. Common apoptosis-detection methods rely on fluorescent markers, which necessitate the use of costly reagents and time-consuming labeling procedures. Label-free methods avoid these problems, but often require specialized instruments instead. Here, we utilize apoptotic-cell disintegration to develop a novel label-free detection method based on the quantification of subcellular debris particles in bright-fieldmicroscopy images. Debris counts show strong correlations with fluorescence-based annexin V staining and can be used to study concentration-dependent and temporal apoptosis activation. The

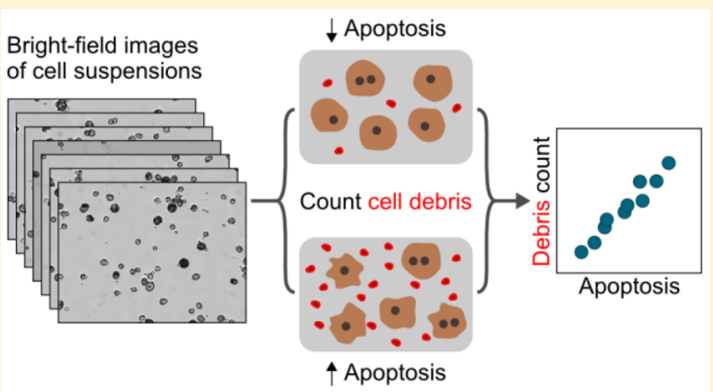
method is rapid, low-cost, and easy to apply, as the only experimental step comprises bright-field imaging of culture-media samples followed by automated image processing. The late-stage nature of the debris measurement means that the method can complement other, established apoptosis assays, and its accessibility will allow a wider community of researchers to study apoptotic cell death.

A poptosis is a highly regulated form of cell death with an important role as a counterpart to mitosis in maintaining physiological homeostasis. ${ }^{1}$ Thus, its dysregulation is associated with various diseases, including cancer and neurodegenerative disorders. ${ }^{2}$ Apoptosis activation is also commonly involved in drug-induced cytotoxicity. ${ }^{3}$ The importance of apoptosis has prompted the development of numerous in vitro detection methods. ${ }^{4}$ Many methods are based on fluorescent labeling of apoptotic features, such as DNA fragmentation (TUNEL), ${ }^{5}$ phosphatidylserine externalization (annexin V binding), ${ }^{6}$ and caspase activation. ${ }^{7}$ However, these methods are usually time-consuming and require expensive reagents and instruments. Furthermore, multiple measurements of the same cells over time are often not possible.

Alternatively, apoptosis can be investigated by observing the distinct morphological changes it entails, typically by light or electron microscopy. ${ }^{8}$ These changes include cell shrinkage, pyknosis, and membrane blebbing and end with fragmentation into membrane-bound vesicles (apoptotic bodies) for removal by phagocytes. ${ }^{9}$ Microscopy-based morphological-detection methods have the advantage of being label-free but are generally qualitative, because of the subjective image interpretation. Nevertheless, there have been attempts to turn morphological observations into quantitative apoptosis assays, using phase-contrast microscopy (PCM) or digitalholographic microscopy (DHM) combined with computational image processing to detect apoptotic changes in cellular shape and volume. ${ }^{10-12}$ These methods have been successfully used to study apoptosis in primary cells and cell lines.
Although PCM- and DHM-based assays seem encouraging, they still require specialized microscopes and complex computational techniques that are not widely available. Other proposed concepts for label-free apoptosis detection (e.g., surface-plasmon resonance, ${ }^{13}$ dielectrophoresis, ${ }^{14}$ and voltammetry ${ }^{15}$ ) involve similar problems. Label-free assays would have a broader appeal if more readily available techniques, such as bright-field microscopy, could be used instead. However, this depends on the identification of apoptotic features that are visible in bright-field microscopes. Apoptotic cell disintegration presents an opportunity here, as apoptotic bodies have diameters of $1-5 \mu \mathrm{m},{ }^{9}$ which is observable at relatively low magnification. Debris particles of this size have been observed after apoptosis induction, but image-based quantification has been difficult. ${ }^{14}$

Here, we use primary human hepatocytes and MDCK cells as models to demonstrate the feasibility of quantifying subcellular debris particles in bright-field-microscopy images as a measure of apoptosis. The method uses a simple ImageJ macro for automated image processing and debris counts, and no special skills are required for its application. The debris we detected were within the size range of apoptotic bodies, and the debris counts were highly correlated with annexin V staining, the most common fluorescent apoptosis marker. Caspase inhibition gave decreased debris counts, consistent

Received: March 9, 2019

Accepted: April 19, 2019

Published: April 19, 2019 
with lower apoptosis levels. Further, we could detect concentration-dependent and temporal apoptosis induction by cytotoxic compounds. Thus, debris counts constitute a novel tool for quantifying late-stage apoptosis in cell culture. Our method is rapid, label-free, and low-cost and should be readily applicable for any laboratory equipped with a light microscope and camera for bright-field imaging.

\section{EXPERIMENTAL SECTION}

Materials. Media and cell-culture supplements were purchased from Thermo Fisher Scientific or Sigma-Aldrich, unless otherwise indicated. Compounds were obtained from Sigma-Aldrich, UBP Bio, and Toronto Research Chemicals.

Human Hepatocytes. Tissue from human-liver resections was obtained, with informed consent, from the Department of Surgery, Uppsala University Hospital, Sweden, as approved by the Uppsala Regional Ethical Review Board (Ethical Approval no. 2009/028). Hepatocytes were isolated using a two-step collagenase-perfusion procedure described elsewhere. ${ }^{16}$ For cryopreservation, hepatocytes were resuspended at $10 \times 10^{6}$ viable cells $/ \mathrm{mL}$ in KaLy-Cell medium (KaLy-Cell) or CryoStor CS10 (BioLife Solutions) with 10\% FBS. Cryovials with $1 \mathrm{~mL}$ cell suspensions were kept in isopropanol freezing containers at $-80{ }^{\circ} \mathrm{C}$ for $2 \mathrm{~h}$ and stored at $-150{ }^{\circ} \mathrm{C}$. Thawing was performed in a $37^{\circ} \mathrm{C}$ water bath for $2 \mathrm{~min}$. The cell suspension was poured into $50 \mathrm{~mL}$ tubes containing prewarmed medium, either DMEM with 30\% isotonic Percoll (GE Healthcare) or cryopreserved-hepatocyte-recovery medium (CHRM, Thermo Fisher Scientific). Tubes were centrifuged at $100 \mathrm{~g}$ for $10 \mathrm{~min}$, and supernatants were aspirated. Hepatocytes were resuspended in warm DMEM with $25 \mathrm{mM}$ HEPES, $4.5 \mathrm{~g} / \mathrm{L}$ glucose, $5 \% \mathrm{FBS}, 4 \mu \mathrm{g} / \mathrm{mL}$ insulin, $1 \mu \mathrm{M}$ dexamethasone, $4 \mathrm{mM} \mathrm{L}-$ glutamine, $100 \mathrm{U} / \mathrm{mL}$ penicillin, and $100 \mu \mathrm{g} / \mathrm{mL}$ streptomycin. For adherent culture, the cells were added to collagen I coated multiwell plates at $0.75 \times 10^{6}$ cells $/ \mathrm{mL}$ and incubated for $3 \mathrm{~h}$ at $37{ }^{\circ} \mathrm{C}$ and $5 \% \mathrm{CO}_{2}$. The medium was then replaced with hepatocyte-maintenance medium (HMM, Lonza) containing $10 \mu \mathrm{g} / \mathrm{mL}$ insulin, $5.5 \mu \mathrm{g} / \mathrm{mL}$ transferrin, $5 \mathrm{ng} / \mathrm{mL}$ selenium, $0.1 \mu \mathrm{M}$ dexamethasone, $100 \mathrm{U} / \mathrm{mL}$ penicillin, and $100 \mu \mathrm{g} / \mathrm{mL}$ streptomycin (complete HMM).

Cell Viability. Cell viability was measured with acridine orange (AO)-propidium iodide (PI) staining using a Cellometer Vision CBA image cytometer (Nexcelom Bioscience). Cell suspensions were gently mixed before collection of $20 \mu \mathrm{L}$ samples for analysis to ensure that representative, homogeneous suspensions were obtained. Fluorescence was analyzed with VB-535-402 (excitation at $475 \mathrm{~nm}$, emission at $535 \mathrm{~nm} ; \mathrm{AO}$ ) and VB-660-502 (excitation at $540 \mathrm{~nm}$, emission at $660 \mathrm{~nm}$; PI) filters.

Apoptosis in Cell Suspensions. Apoptosis in hepatocyte suspensions was analyzed with fluorescent markers for activated caspase-3 and -8 using CaspGLOW Fluorescein Active Caspase Staining Kits (BioVision) and an annexin VFITC conjugate (BioLegend) according to the manufacturer's instructions, with minor modifications. Samples were gently mixed, and $20 \mu \mathrm{L}$ was collected from each, which was analyzed with a Cellometer Vision CBA, using the VB-535-402 filter.

Cell-Debris Analysis. Using the open-source ImageJ distribution Fiji, ${ }^{17,18}$ we developed an automated macro for counting debris particles in bright-field images. Images were acquired with the built-in microscope of the Cellometer Vision CBA (10× magnification), in parallel with fluorescence analysis. First, images were converted to 8 bit grayscale.
Second, Gray Morphology was applied, using the "erode" operator and a 5 pixel circular-structure-element radius. Third, Subtract Background was used with the "light background" and "sliding paraboloid" options active, using an 8 pixel rolling-ball radius. Fourth, images were automatically thresholded with the Triangle algorithm. Fifth, a watershed operation was performed. Finally, Analyze Particles was used to count particles of 10-200 square pixels with circularity of $0.5-1$. Data were obtained as a tab-separated text file. Samples were counted in technical quadruplicates. The operations and size settings used were well suited for the cells we analyzed in this particular imaging setup, but optimization might be required for other cells and microscopes. The ImageJ macro is provided as Supporting Information, together with more detailed usage instructions. A slightly modified macro was used to measure particle sizes. Notably, this did not include the Gray Morphology and Subtract Background operations, which facilitate detection but alter particle size and shape.

Collection of Nonattached Hepatocytes. Hepatocytes were cultured for $3 \mathrm{~h}$. Nonattached hepatocytes in culture medium were carefully collected to obtain representative samples, centrifuged at $150 \mathrm{~g}$ for $5 \mathrm{~min}$, and resuspended in a small volume of PBS. Bright-field images of $20 \mu \mathrm{L}$ samples were obtained as in the Cell-Debris Analysis section.

Apoptosis Inhibition in Cultured Hepatocytes. Hepatocytes were cultured with a concentration series of the pancaspase inhibitor Z-VAD-FMK $(0-190 \mu \mathrm{M})$. The culture medium was carefully collected after $3 \mathrm{~h}$, and bright-field images of $20 \mu \mathrm{L}$ cell-suspension samples were obtained as in the Cell-Debris Analysis section.

Apoptosis in Hepatocyte Suspensions. Hepatocytes were resuspended in complete HMM and diluted to $1.5 \times 10^{6}$ cells/mL; $500 \mu \mathrm{L}$ of cell suspension was transferred to $10 \mathrm{~mL}$ tubes and gently rocked at $37{ }^{\circ} \mathrm{C}$. Annexin V staining and debris counts were analyzed in representative samples after 0 , 2, 4, and $7 \mathrm{~h}$.

Apoptosis Induction in Hepatocytes and MDCK Cells. Hepatocytes were cultured in collagen I coated 24-well plates (375 000 cells/well). MDCK cells were suspended in DMEM with GlutaMax and 10\% FBS, seeded in 96-well plates $(75000$ cells/well), and incubated for $24 \mathrm{~h}$ at $37{ }^{\circ} \mathrm{C}$ and $5 \% \mathrm{CO}_{2}$. The medium was replaced, and compounds were added from DMSO stocks. Representative samples were collected after 7, 24 , and 48 h by carefully pipetting up and down several times. Debris counts were performed, and caspase activity was measured using the CellEvent Caspase-3/7 Green Detection Reagent (Thermo Fisher Scientific), according to the manufacturer's instructions.

\section{RESULTS AND DISCUSSION}

Measurements of Cell Debris in Bright-Field-Microscopy Images. We routinely acquire bright-field-microscopy images of human-hepatocyte suspensions together with cellconcentration and -viability assessments. In these, we have observed that less-viable cell preparations tend to contain higher levels of debris particles. This prompted an investigation into the utility of debris quantification as a simple measure of apoptosis. Primary human hepatocytes are widely used in studies of the absorption, distribution, metabolism, excretion, and toxicity (ADMET) of xenobiotics, and the ability to probe different apoptosis stages in vitro is important. ${ }^{19}$ Thus, we developed an ImageJ macro for automatic image processing and debris counting (Figure 1). Visual inspection confirmed 
that debris particles were successfully identified and counted and that cells were not included in the counts (Figure S1).

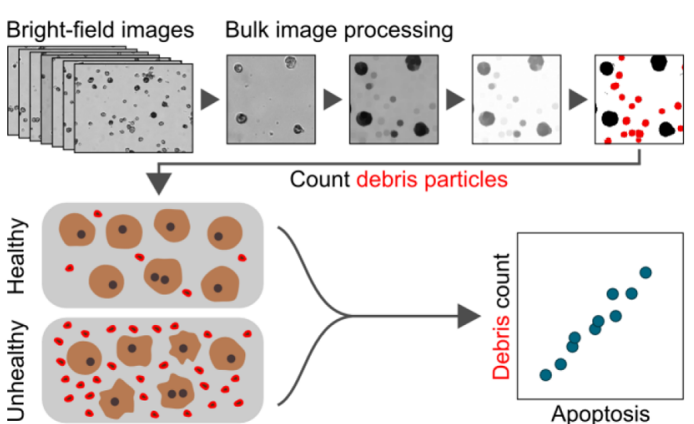

Figure 1. Quantification of subcellular debris using bright-fieldmicroscopy images. Images were processed with an ImageJ macro, and debris particles in the size range of apoptotic bodies were automatically counted (highlighted in red).

Cell Debris as a Quantitative Measure of Apoptosis. To investigate the origins of the imaged debris particles, we used a modified macro (see the Experimental Section) to measure actual particle-size distributions in cryopreserved human-hepatocyte batches from 14 different donors immediately after thawing. The mean particle diameter was $4.4 \pm 2.2$ $\mu \mathrm{m}$, with approximately $70 \%$ of the particles within $2-5 \mu \mathrm{m}$ (Figure 2a). This matches reported sizes of apoptotic bodies, ${ }^{9}$ indicating that the majority of the detected debris originated from apoptosis. In fact, the small debris particles associated with total plasma-membrane rupture caused by necrotic cell death $^{20}$ are likely not visible in our low-magnification images. Particles larger than $5 \mu \mathrm{m}$ presumably comprised aggregates and, possibly, residual small cells, such as erythrocytes and nonparenchymal liver cells.

We then evaluated the applicability of debris quantification for measuring apoptosis by comparing post-thaw debris counts from the 14 hepatocyte batches with different fluorescent apoptosis markers. We used image cytometry for these analyses, which has been shown to give similar results to conventional flow cytometry. ${ }^{21}$ As expected, debris counts were negatively correlated with general cell viability (Pearson's $r=-0.70$, Figure S2a). However, a much stronger correlation was observed with annexin $\mathrm{V}$ staining of phosphatidylserine externalization (Pearson's $r=0.91$, Figure $2 \mathrm{~b}$ ), a widely used apoptosis marker. ${ }^{4,22}$ Interestingly, debris counts covered a wider range of values than annexin $\mathrm{V}$ staining, with maximum and minimum fold differences of 3.5 and 2.1, respectively, showing that the method can measure a continuous spectrum of apoptosis levels.

In contrast, we observed only moderate correlations with markers for caspase-8 (initiator caspase) and caspase-3 (effector caspase) activation (Pearson's $r=0.61$ and 0.59, respectively; Figure $S 2 b, c)$. This is consistent with the later occurrence of phosphatidylserine externalization compared with caspase activation during apoptosis progression and with cell disintegration constituting the last stage (Figure 2c). ${ }^{9,23}$ Indeed, the early markers (caspase- 8 and caspase- 3 ) showed strong correlations with each other, as did the late markers (annexin V and debris counts), but the correlations between early and late markers were much weaker (Figures $2 \mathrm{~d}$ and S2d-f). Altogether, these results demonstrate the utility of counting cell debris to quantify the total levels of late-stage apoptosis in a cell sample and that the applicability of the method is not hampered by the relatively minor presence of particles that may have nonapoptotic origins.

Reduction of Cell Debris via Apoptosis Inhibition. To further investigate the utility of debris quantification, we applied the method to images of cryopreserved hepatocyte suspensions from 41 different experiments, including images captured immediately after thawing and images of the corresponding culture medium after $3 \mathrm{~h}$ in collagen I coated multiwell plates (containing nonattached cells). We have previously demonstrated that nonattached hepatocytes show increased apoptosis activation. ${ }^{24}$ Here, we observed that this was accompanied by increased debris counts, with 2 -fold higher levels in nonattached cell fractions than in cell suspensions immediately after thawing (Figure 3a).

Next, we investigated whether apoptosis inhibition would decrease debris counts. For this purpose, cells from four hepatocyte batches of different quality (in terms of attachment ability and cellular morphology) ${ }^{24}$ were cultured for $3 \mathrm{~h}$ with 0-190 $\mu \mathrm{M}$ Z-VAD-FMK, a potent pan-caspase inhibitor. ${ }^{25}$ Debris counts in the culture medium decreased with increasing $\mathrm{Z}$-VAD-FMK concentration in a dose-dependent manner for all except the highest-quality batch, where almost all cells could attach and survive (Figure $3 \mathrm{~b}$ ). This further supported the notion that the observed debris mainly resulted from apoptosis.

Detachment-Induced Apoptosis in Hepatocytes. By necessity, hepatocyte isolation disrupts cell-cell and cellmatrix connections. The loss of anchorage triggers apoptosis
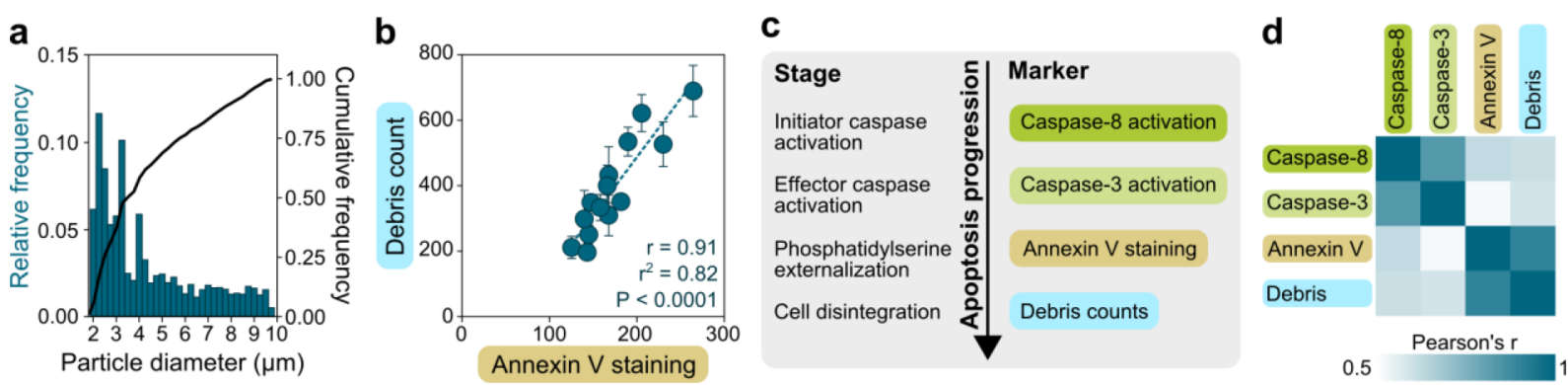

Figure 2. Quantification of apoptosis levels by debris counts. (a) Size distribution of debris particles in 14 cryopreserved human-hepatocyte batches immediately after thawing, shown as relative and cumulative frequencies (represented by the bars and line, respectively). (b) Batch-specific debris counts in the 14 batches correlated with annexin V staining of phosphatidylserine externalization. Error bars show standard deviations of technical quadruplicates. Annexin V staining is represented as mean fluorescence intensity per cell, normalized by cell size. (c) Apoptotic stages measured by the different markers used here. (d) Correlations of all four apoptosis markers used in this analysis. 

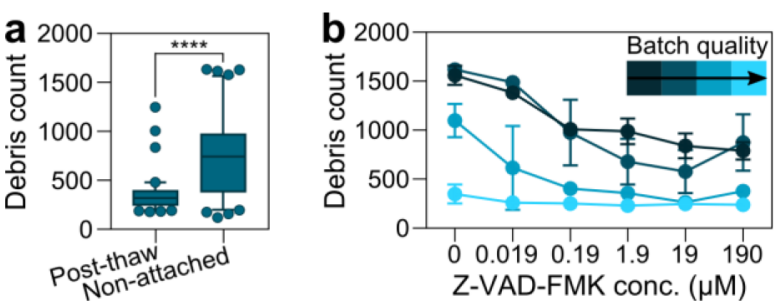

Figure 3. Debris reduction by caspase inhibition. (a) Debris counts from 41 different hepatocyte experiments, both immediately after thawing and in the nonattached fraction after $3 \mathrm{~h}$ in culture. Boxes range between the 25th and 75th percentiles, lines show medians, and whiskers denote the 10th and 90th percentiles. $* * * * P<0.0001$, Student's $t$ test. (b) Debris counts in the nonattached fractions of four hepatocyte batches treated with the apoptosis inhibitor Z-VAD-FMK. Colors indicate batch quality regarding attachment ability and cellular morphology in adherent culture. Values are shown as means and standard deviations of two independent experiments.

through anoikis. ${ }^{26}$ Such spontaneous apoptosis progresses relatively rapidly in suspension culture. ${ }^{27}$ Therefore, we assessed whether hepatocytes kept in suspension would show a temporal increase in debris and apoptosis levels. Hepatocytes were cultured at $37{ }^{\circ} \mathrm{C}$ for $7 \mathrm{~h}$ and analyzed with annexin $\mathrm{V}$ staining and debris counts. Both parameters showed similar continuous increases over this period (Figure 4), demonstrat-

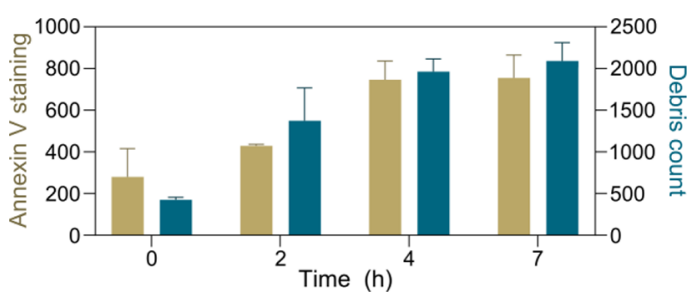

Figure 4. Temporal apoptosis induction in hepatocyte suspensions. Apoptosis was analyzed by annexin $\mathrm{V}$ staining and debris counts. Annexin $\mathrm{V}$ staining is represented as mean fluorescence intensity per cell, normalized by cell size. Values are shown as means and standard deviations of two independent experiments.

ing that debris counts can be used to follow apoptosis over time. The fact that debris gradually builds up in the culture medium presents an interesting advantage of our method. Apoptosis can proceed from initiation to completion in only $2-3 \mathrm{~h},{ }^{1}$ and methods based on intermediate steps of the apoptotic process would not detect completely disintegrated cells if performed too late, thus potentially underestimating apoptosis levels in cases of very rapid progression. Contrarily, debris counts provide a measure of total apoptosis levels up until the time of imaging. We note that our method can thus not be used to follow the time-course of apoptosis in individual cells, but it enables the study of overall apoptosis progression in the same culture vessel over time.

Compound-Induced Apoptosis in Hepatocytes and the MDCK Cell Line. Finally, we investigated whether debris counts could be used to measure concentration-dependent apoptosis upon the addition of toxic compounds. We tested primary human hepatocytes as well as Madin-Darby caninekidney (MDCK) cells, a canine cell line originating from the kidney tubular epithelium, to assess differences in the responses of two principally different cell types. Further, hepatocytes and MDCK cells are important for studies of hepatotoxicity and nephrotoxicity, respectively, ${ }^{19,28}$ highlight- ing the relevance of apoptosis measurements in these cell types. After $24 \mathrm{~h}$ in culture, two concentrations of celecoxib (20 and $100 \mu \mathrm{M})$, chlorpromazine (10 and $50 \mu \mathrm{M})$, diclofenac (100 and $500 \mu \mathrm{M})$, and doxorubicin (10 and $50 \mu \mathrm{M})$ were added. Concentrations were selected on the basis of previously observed toxicity. ${ }^{29-32}$ Apoptosis induction was monitored by caspase activity and debris counts after 7, 24, and $48 \mathrm{~h}$.

Celecoxib, a known apoptosis inducer, ${ }^{29}$ triggered a sharp concentration-dependent increase in caspase activity already after $7 \mathrm{~h}$ in both cell types (Figure 5a). This was reflected by

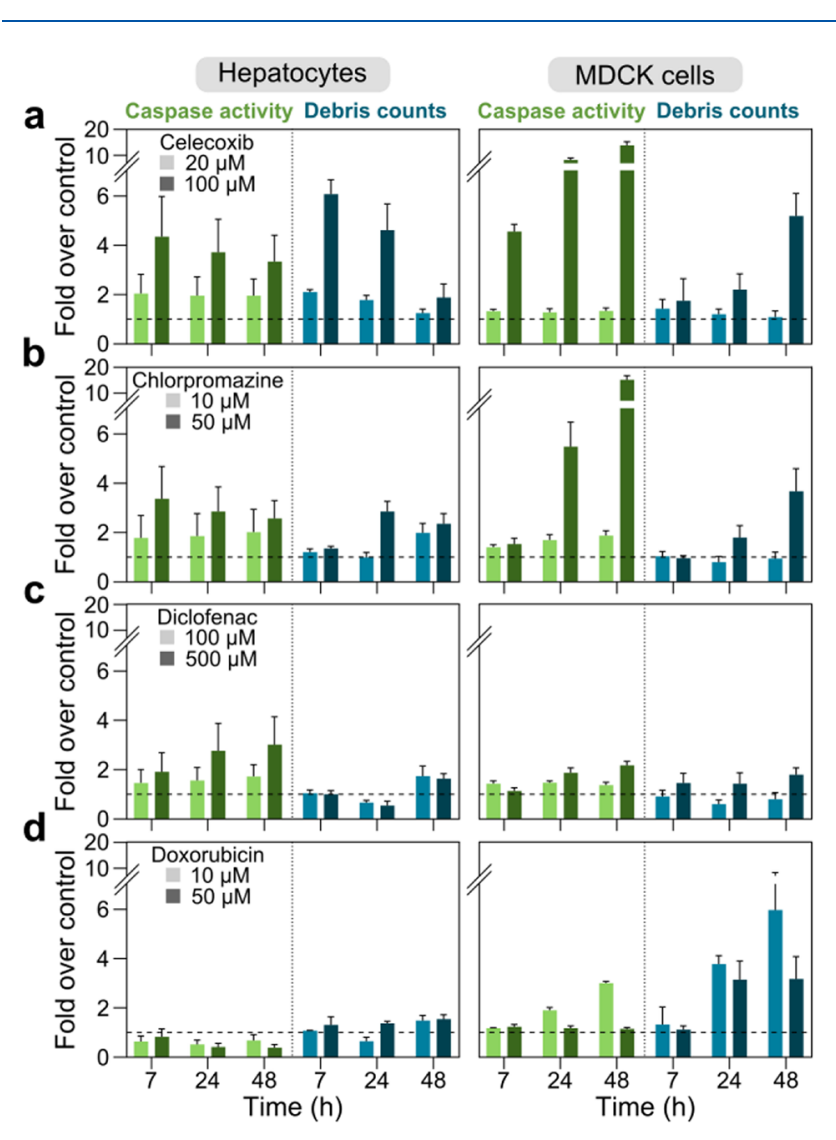

Figure 5. Apoptosis induction in adherent cultures of human hepatocytes and MDCK cells, analyzed by caspase activity and debris counts. Two concentrations were tested of (a) celecoxib, (b) chlorpromazine, (c) diclofenac, and (d) doxorubicin. Values are shown as means and standard deviations of two independent experiments.

early high debris counts in hepatocytes, whereas MDCK cells showed a slower increase up to $48 \mathrm{~h}$. Celecoxib-treated hepatocytes showed markedly declined fold increases compared with the untreated control after $48 \mathrm{~h}$, which may point to late breakdown of rapidly formed debris. Indeed, it is known that apoptotic bodies may eventually rupture in the absence of phagocytosis. ${ }^{20}$ Chlorpromazine triggered slightly lower levels of caspase activation in hepatocytes, with no debris until after $24 \mathrm{~h}$ (Figure 5b). Both parameters gradually increased after 24 $\mathrm{h}$ of chlorpromazine exposure in MDCK cells. Diclofenac gave a temporal increase in hepatocyte caspase activity, with a considerable lag time before the initial appearance of debris at $48 \mathrm{~h}$ (Figure 5c). MDCK cells were relatively unaffected by diclofenac. This discrepancy likely results from the formation of diclofenac metabolites in hepatocytes, which are more toxic than the compound itself. ${ }^{31}$ Doxorubicin did not trigger 
caspase activation in hepatocytes and only led to slightly increased debris levels (Figure $5 \mathrm{~d}$ ). In contrast, doxorubicin increased caspase activity in MDCK cells and resulted in very high debris counts. The higher apoptosis induction in the cell line is probably related to the mechanism of action of doxorubicin, which involves DNA-binding and antiproliferation. ${ }^{33}$ Human hepatocytes do not proliferate in vitro, ${ }^{34}$ which could partly explain their decreased sensitivity. Curiously, doxorubicin gave higher levels of caspase activation and debris counts in MDCK cells at the low concentration $(10 \mu \mathrm{M})$, possibly because of extreme toxicity at the high concentration (50 $\mu \mathrm{M})$, which may have killed the cells through other mechanisms before caspase activation could be detected.

\section{CONCLUSIONS}

In this study, we show that subcellular debris particles visible under a bright-field microscope can be used to quantitatively measure late-stage apoptosis levels. The method we developed is experimentally straightforward, as it only entails bright-field imaging of culture-media samples and automated image processing and can be used to measure apoptosis levels in suspension and adherent cell culture. Because no expensive reagents or specialized instruments are required, the method enables label-free detection of late-stage apoptosis in laboratories where other approaches would be prohibitively costly and ultimately allows a broader range of researchers to study this important mechanism of cellular demise.

\section{ASSOCIATED CONTENT}

\section{S Supporting Information}

The Supporting Information is available free of charge on the ACS Publications website at DOI: 10.1021/acs.analchem.9b01243.

Detailed usage instructions for the debris-counting macro, typical bright-field images of human hepatocytes in suspension showing all processing steps of the debriscounting macro, and comparisons of debris counts and other apoptosis markers in 14 human-hepatocyte batches (PDF)

ImageJ macro for debris counting (ZIP)

\section{AUTHOR INFORMATION}

\section{Corresponding Author}

*E-mail: per.artursson@farmaci.uu.se. Tel.: +46-184714471.

ORCID $\odot$

Magnus Ölander: 0000-0002-4502-8184

Notes

The authors declare no competing financial interest.

\section{ACKNOWLEDGMENTS}

We thank Maria Karlgren for providing the MDCK cells. This work was supported by the Swedish Research Council (grant nos. 2822 and 01951).

\section{REFERENCES}

(1) Elmore, S. Toxicol. Pathol. 2007, 35, 495-516.

(2) Thompson, C. B. Science 1995, 267, 1456-1462.

(3) Liebler, D. C.; Guengerich, F. P. Nat. Rev. Drug Discovery 2005, 4, 410 .

(4) Martinez, M. M.; Reif, R. D.; Pappas, D. Anal. Methods 2010, 2, 996-1004.
(5) Gavrieli, Y.; Sherman, Y.; Ben-Sasson, S. A. J. Cell Biol. 1992, 119, 493-501.

(6) Koopman, G.; Reutelingsperger, C.; Kuijten, G.; Keehnen, R.; Pals, S.; Van Oers, M. Blood 1994, 84, 1415-1420.

(7) Gurtu, V.; Kain, S. R.; Zhang, G. Anal. Biochem. 1997, 251, $98-$ 102.

(8) Häcker, G. Cell Tissue Res. 2000, 301, 5-17.

(9) Atkin-Smith, G. K.; Poon, I. K. Trends Cell Biol. 2017, 27, 151162.

(10) Aftab, O.; Nazir, M.; Fryknäs, M.; Hammerling, U.; Larsson, R.; Gustafsson, M. G. Apoptosis 2014, 19, 1411-1418.

(11) Kemmler, M.; Fratz, M.; Giel, D. M.; Saum, N.; Brandenburg, A.; Hoffmann, C. J. Biomed. Opt. 2007, 12, 064002.

(12) Khmaladze, A.; Matz, R. L.; Epstein, T.; Jasensky, J.; Holl, M. M. B.; Chen, Z. J. Struct. Biol. 2012, 178, 270-278.

(13) Maltais, J.-S.; Denault, J.-B.; Gendron, L.; Grandbois, M. Apoptosis 2012, 17, 916-925.

(14) Henslee, E. A.; Serrano, R. M. T.; Labeed, F. H.; Jabr, R. I.; Fry, C. H.; Hughes, M. P.; Hoettges, K. F. Analyst 2016, 141, 6408-6415.

(15) Takano, S.; Shiomoto, S.; Inoue, K. Y.; Ino, K.; Shiku, H.; Matsue, T. Anal. Chem. 2014, 86, 4723-4728.

(16) LeCluyse, E. L.; Alexandre, E. Isolation and Culture of Primary Hepatocytes from Resected Human Liver Tissue. In Hepatocytes: Methods and Protocols; Maurel, P., Ed.; Methods in Molecular Biology; Humana Press, 2010; pp 57-82.

(17) Schneider, C. A.; Rasband, W. S.; Eliceiri, K. W. Nat. Methods 2012, 9, 671 .

(18) Schindelin, J.; Arganda-Carreras, I.; Frise, E.; Kaynig, V.; Longair, M.; Pietzsch, T.; Preibisch, S.; Rueden, C.; Saalfeld, S.; Schmid, B.; et al. Nat. Methods 2012, 9, 676.

(19) Godoy, P.; Hewitt, N. J.; Albrecht, U.; Andersen, M. E.; Ansari, N.; Bhattacharya, S.; Bode, J. G.; Bolleyn, J.; Borner, C.; Boettger, J.; et al. Arch. Toxicol. 2013, 87, 1315-1530.

(20) Fink, S. L.; Cookson, B. T. Infect. Immun. 2005, 73, 19071916.

(21) Chan, L. L.; Lai, N.; Wang, E.; Smith, T.; Yang, X.; Lin, B. Apoptosis 2011, 16, 1295-1303.

(22) van Engeland, M.; Nieland, L. J.; Ramaekers, F. C.; Schutte, B.; Reutelingsperger, C. P. Cytometry 1998, 31, 1-9.

(23) Nagata, S.; Hanayama, R.; Kawane, K. Cell 2010, 140, 619630.

(24) Ölander, M.; Wiśniewski, J. R.; Flörkemeier, I.; Handin, N.; Urdzik, J.; Artursson, P. Arch. Toxicol. 2019, 93, 819-829.

(25) Garcia-Calvo, M.; Peterson, E. P.; Leiting, B.; Ruel, R.; Nicholson, D. W.; Thornberry, N. A. J. Biol. Chem. 1998, 273, 32608-32613.

(26) Frisch, S. M.; Francis, H. J. Cell Biol. 1994, 124, 619-626.

(27) Smets, F. N.; Chen, Y.; Wang, L.-J.; Soriano, H. E. Mol. Genet. Metab. 2002, 75, 344-352.

(28) Pfaller, W.; Gstraunthaler, G. Environ. Health Perspect. 1998, 106, 559-569.

(29) Kern, M. A.; Haugg, A. M.; Koch, A. F.; Schilling, T.; Breuhahn, K.; Walczak, H.; Fleischer, B.; Trautwein, C.; Michalski, C.; SchulzeBergkamen, H.; et al. Cancer Res. 2006, 66, 7059-7066.

(30) Bell, C. C.; Lauschke, V. M.; Vorrink, S. U.; Palmgren, H.; Duffin, R.; Andersson, T. B.; Ingelman-Sundberg, M. Drug Metab. Dispos. 2017, 45, 419.

(31) Gómez-Lechón, M. J.; Ponsoda, X.; O'connor, E.; Donato, T.; Castell, J. V.; Jover, R. Biochem. Pharmacol. 2003, 66, 2155-2167.

(32) Green, P. S.; Leeuwenburgh, C. Biochim. Biophys. Acta, Mol. Basis Dis. 2002, 1588, 94-101.

(33) Tacar, O.; Sriamornsak, P.; Dass, C. R. J. Pharm. Pharmacol. 2013, 65, 157-170.

(34) Shan, J.; Schwartz, R. E.; Ross, N. T.; Logan, D. J.; Thomas, D.; Duncan, S. A.; North, T. E.; Goessling, W.; Carpenter, A. E.; Bhatia, S. N. Nat. Chem. Biol. 2013, 9, 514-520. 